\title{
Involvement of platelet glycoprotein Ib in platelet microparticle mediated neutrophil activation
}

\author{
Shyh-Chyi Lo ${ }^{1,2}$, Ching-Yu Hung ${ }^{1}$, Dong-Tsamn Lin $^{2}$, Hui-Chin Peng ${ }^{1} \&$ \\ Tur-Fu Huang ${ }^{1, *}$ \\ ${ }^{1}$ Department of Pharmacology, College of Medicine, National Taiwan University, No. 1, Jen-Ai Rd., Sec. 1, \\ Taipei, Taiwan; ${ }^{2}$ Department of Laboratory Medicine, National Taiwan University Hospital, No. 7 Chung- \\ Shan S Rd., 100, Taipei, Taiwan, ROC
}

Received 16 April 2006; accepted 17 July 2006

(C) 2006 National Science Council, Taipei

Key words: glycoprotein Ib antagonist, leukocyte, microparticle, platelet

\begin{abstract}
Summary
Platelet microparticles (MPs) are membrane vesicles shed by platelets after activation, and carry antigens characteristic of intact platelets, such as glycoprotein (GP) IIb/IIIa, GPIb and P-selectin. Elevated platelet MPs have been observed in many disorders in which platelet activation is documented. Recently, platelet GPIb has been implicated in the mediation of platelet-leukocyte interaction via binding to its ligand Mac-1 on leukocyte. The role of GPIb for mediating adhesion-activation interactions between platelet MPs and leukocytes has not been clarified. In this study we investigate the role of GPIb in the interplay between platelet MPs and neutrophils. Platelet MPs were obtained from collagen-stimulated platelet-rich plasma (PRP). In a study model of neutrophil aggregation, platelet MPs can serve a bridge to support neutrophil aggregation under venous level shear stress, suggesting that platelet MPs may enhance leukocyte aggregation, which would bear clinical relevance in diseases where the platelet MPs are elevated. The level of aggregation can be reduced by GPIb blocking antibodies, AP1 and SZ2, but not by anti-CD18 mAb. The GPIb blocking antibodies also decreased platelet MP-mediated neutrophil activation, including $\beta 2$ integrin expression, adherence-dependent superoxide release and platelet MP-mediated neutrophil adherence to immobilized fibrinogen. Our data provide the evidence for the involvement of GPIb-Mac-1 interaction in the cross-talk between platelet MPs and neutrophils.
\end{abstract}

\section{Introduction}

Upon activation, leukocytes respond with degranulation, increased respiratory bursts, chemotaxis, and phagocytosis. All of these processes may be influenced by platelets. For instance, interaction between neutrophils and platelets elicits interleukin-8 secretion [1] and oxidative burst of neutrophils [2]. Platelet-related adenine nucleotides [3]

*To whom correspondence should be addressed. Fax: + 886-223417930; E-mail: turfu@ccms.ntu.edu.tw and platelet-derived growth factor (PDGF) may induce leukocyte degranulation. Adherent platelet and platelet-released substances, such as PDGF [4], platelet factor 4 [5], and thromboxane A2 (TXA2) [6], may enhance leukocyte rolling and adherence to the vessel wall. Thus, platelets and platelet-derived soluble products may influence leukocyte function in a complex manner.

During the inflammation process, the interaction of neutrophils with activated platelets is coordinated by an adhesion cascade [7] in which platelet $\mathrm{P}$-selectin binds to $\mathrm{P}$-selectin glycoprotein 
ligand-1 (PSGL-1) on leukocytes to promote the initial tethering of the cells and the subsequent firm adhesion is mediated by $\beta 2$ integrin CD11b/ 18 (Mac-1) binding to either platelet-GPIb [8] or to fibrinogen bound to platelet GPIIb/IIIa or integrin $\alpha \mathrm{v} \beta 3$ [9].

Adhesion between platelets and leukocytes may represent an important process in hemostasis and thrombosis. Thrombotic stimuli induce platelets to aggregate via activation of GPIIb/ IIIa and to express P-selectin on their surfaces at sites of vascular injury [10]. Under normal conditions, up-regulated P-selectin mediates leukocyte accumulation and fibrin formation [11] and accelerates clotting. However, under pathological conditions, these adhesive interactions may promote thrombosis and vascular occlusion, thereby impairing blood flow and exacerbating ischemia.

Platelet activation is known to result in the release of microparticles by shedding of membranes from the surface [7, 12]. Platelet MPs expose platelet-specific antigens on their surface and can adhere to leukocyte in a P-selectin dependent manner [7]. Clinical studies have shown that elevated levels of microparticles were found in patients with disseminated intravascular coagulation [13], unstable angina [14], myocardial infarction $[15,16]$, coronary angiography [17], transient ischemia attacks [18], and diabetes mellitus [19]. A deficiency of platelet MP generation can result in a bleeding disorder characterized with prolonged bleeding time [20].

Recently, Simon et al. reported that platelet GPIb $\alpha$ is the counter-receptor for Mac-1 on leukocyte, accounting for firm adhesion and trans-platelet migration of leukocytes on vascular thrombus [8]. It has been previously reported that platelet MP expressed less P-selectin than platelet and the interaction between platelet MP and neutrophil is somehow different from plateletneutrophil interaction with regard to P-selectin and sialyl Lewis inhibitory studies [21]. However, the role of GPIb $\alpha$ in the interplay between platelet MP and leukocyte has not been addressed. To our knowledge, few papers have addressed the role of platelet GPIb in plateletMP-neutrophil interaction. In this study, we intend to explore how platelet-GPIb $\alpha$ inhibitors affect the interaction between platelet MP and neutrophil.

\section{Methods and materials}

\section{Antibodies and reagents}

The P-selectin-blocking antibody (mAb) 9E1 was purchased from R\&D System (Minneapolis, $\mathrm{MN}$, USA). Anti-GPIb $\alpha \mathrm{mAb} \mathrm{SZ2}$, and antiPSGL-1 mAb PL1 and non-immune IgG were from Immunotech (France). The anti-GPIIb/IIIa chimeric 7E3 Fab (ReoPro) was a gift kindly provided by Lilly Crop., Taiwan. Anti-GPIb $\alpha$ Ab AP1 was provided by Dr. Robert Montgomery (Milwaukee Blood Center, Milwaukee, WI, USA). The $\beta 2$-integrin (CD18) blocking mAb (clone TS1/18) was purchased from Endogen (Woburn, MA, USA). FITC-conjugated anti-CD42a (platelet GPIX) mAb (clone ALMA.16), FITC-conjugated anti-CD62P (P-selectin) mAb (clone AK-4), PE conjugated anti-CD14 mAb (clone M5E2) and PE-conjugated anti-CD11b mAb (clone M1/70) were purchased from Becton Dickinson (San Diego, CA, USA). Formyl-Met-Leu-Phe (fMLP) was purchased from Sigma Chemical (St. Louis, MO, USA). The fluorescent dye, 2'-7'-bis(2-carboxyethyl)-5(6)-carboxyfluorescein (BCECF-AM) was from Molecular Probe (Eugene, OR, USA). Triflavin, an Arg-Gly-Asp (RGD)-containing snake venom peptide, was prepared as previously reported $[22,23]$.

\section{Preparation of neutrophils, and platelet-rich plasma}

Venous blood was obtained from healthy volunteers by clean venipuncture of an antecubital vein and collected in sterile polypropylene tubes containing $1 / 10$ volume of $3.2 \%$ sodium citrate. Blood samples were centrifuged at room temperature for $10 \mathrm{~min}$ at $240 \times g$ to obtain platelet-rich plasma (PRP). The sediments obtained after centrifugation and removal of PRP were kept at room temperature for the study of whole blood reconstitution (see below). For separation of neutrophils, the sediments were first diluted 1:1 part of PBS, and then were sedimented with $3 \%$ dextran-saline at room temperature. The upper leukocyte-enriched plasma was collected and separated by differential centrifugation over a Ficoll-Hypaque gradient. After hypotonic lysis of erythrocytes, isolated neutrophils were washed twice with normal saline and maintained in ice-cold Hanks' HEPES buffer (Hanks' balanced salt solution, 
$0.5 \%$ bovine serum albumin (BSA), $10 \mathrm{mM} \mathrm{HE}$ PES, $1 \mathrm{mM} \mathrm{CaCl}, 1 \mathrm{mM} \mathrm{MgCl}_{2}$, pH 7.4). More than $95 \%$ of cells were viable as determined by trypan blue exclusion.

\section{Platelet-derived microparticles preparation}

Platelet-derived MP were prepared by incubating PRP with $10 \mu \mathrm{g} / \mathrm{ml}$ collagen for up to $1 \mathrm{~h}$ at $37^{\circ} \mathrm{C}$ and the collagen-treated PRP was centrifuged for $15 \mathrm{~min}$ at $5000 \mathrm{~g}$. The supernatants were collected as platelet MP-containing plasma. In some study, platelet MP-containing plasma was further centrifuged for $30 \mathrm{~min}$ at $17,500 \mathrm{~g}$, the obtained supernatant would serve as MP-free control for confirmation of the MP-induced effects.

\section{Neutrophil aggregation supported by platelet MP}

Homotypic neutrophil aggregation study was performed in accordance with the technique of Tseng et al. [24] with modifications. For study of homotypic aggregation induced by IMLP, neutrophils $\left(5 \times 10^{6} / \mathrm{ml}\right.$, suspended in Hanks' HEPES buffer $)$ were first treated with various inhibitors at $37{ }^{\circ} \mathrm{C}$ for $10 \mathrm{~min}$ before the addition of fMLP. In the study of platelet MP-supported neutrophil aggregation, neutrophils $\left(5 \times 10^{6} / \mathrm{ml}\right)$ were mixed with platelet MP-containing plasma in $1: 1 \mathrm{v} / \mathrm{v}$ in the presence or absence of various inhibitors. Neutrophils were then sheared in a Payton aggregometer $(700 \mathrm{rpm})$ at $37^{\circ} \mathrm{C}$ for $15 \mathrm{~min}$ followed by fixation with $2 \%$ glutaraldehyde. The average shear rate was estimated at about $100 \mathrm{~s}^{-1}$. However, much higher shear rates are present at the surface of the rotating bar [25]. The flow cytometric detection of neutrophil aggregation was measured as previously described [24]. Neutrophils were identified by means of forward and side scatter. Single neutrophils were defined by proper adjustment of the autofluorescence, and aggregates were resolved as integral multiples of the singlet fluorescence channel, as shown in Figure 1A. The ratio of neutrophils in aggregate to the total number of neutrophils was calculated by use of the formula, $\%$ aggregation $=\left(2 \mathrm{D}+3 \mathrm{~T}+4 \mathrm{Q}^{+}\right) /(\mathrm{S}+2 \mathrm{D}+$ $3 \mathrm{~T}+4 \mathrm{Q}^{+}$), where $\mathrm{S}$ represents singlet neutrophils and the neutrophil aggregate sizes are given as $\mathrm{D}$ (doublets), $\mathrm{T}$ (triplets), and $\mathrm{Q}^{+}$(quartets and larger unresolved neutrophil aggregates) [26, 27].

\section{Adhesion assay}

Adhesion assays for neutrophil binding to immobilized fibrinogen were performed as previously described with some modifications [28]. Neutrophils were incubated with BCECF-AM $(0.2 \mathrm{M})$ at $37^{\circ} \mathrm{C}$ for $30 \mathrm{~min}$ followed by washing twice with PBS and then suspended in Hanks' HEPES buffer. In the adhesion inhibition study, chemoattractantor platelet MP-stimulated neutrophils were incubated with various inhibitors. After $15 \mathrm{~min}$ incubation at $37^{\circ} \mathrm{C}, 1 \times 10^{5}$ neutrophils were seeded into wells which had been coated with human fibrinogen $(10 \mu \mathrm{g})$ for $30 \mathrm{~min}$ at $37^{\circ} \mathrm{C}$. After incubation for $1 \mathrm{~h}$ at $37^{\circ} \mathrm{C}$, unbound cells were removed by gentle washing. Attached cells were solubilized in $0.1 \%$ Triton $\mathrm{X}-100$, and the fluorescence emission at $530 \mathrm{~nm}$ was read on a Cytofluor microplate reader. The adhesion of neutrophils was quantified as the percentage of total loaded cells measured before washing. Nonspecific attachment was determined in the presence of $5 \mathrm{mM}$ EDTA. The results are expressed as mean $\pm \mathrm{SD}$.

\section{Adhesion-dependent superoxide release by neutrophils}

Superoxide production by adherent neutrophils was determined by the cytochrome $c$ reduction method [28]. Ninety-six flat-bottomed microplates were precoated with fibrinogen. Neutrophils $\left(2 \times 10^{5}\right)$ pretreated with various inhibitors for $15 \mathrm{~min}$ at $37^{\circ} \mathrm{C}$ were added to wells in the presence of $1 \mu \mathrm{M}$ fMLP or $50 \mu 1$ platelet MPs in the presence of $0.5 \mathrm{mg} / \mathrm{ml}$ cytochrome $c$. Neutrophils were allowed to adhere up to $60 \mathrm{~min}$ in a $5 \% \mathrm{CO}_{2}$ atmosphere at $37{ }^{\circ} \mathrm{C}$. Plates were read using an ELISA reader (Bio-Tek Instruments, USA) to quantify the reduction of cytochrome $c$ at $550 \mathrm{~nm}$. Type I superdismutase (SOD, at $20 \mathrm{U}$ ) was added to confirm that the reduction of cytochrome $c$ was caused by superoxide production. Results were expressed as arbitrary units.

\section{Flow cytometric study of the reconstituted blood samples}

We prepared the reconstituted blood samples according to the procedure described by Scholz et al. [29] with some modifications. The cell 

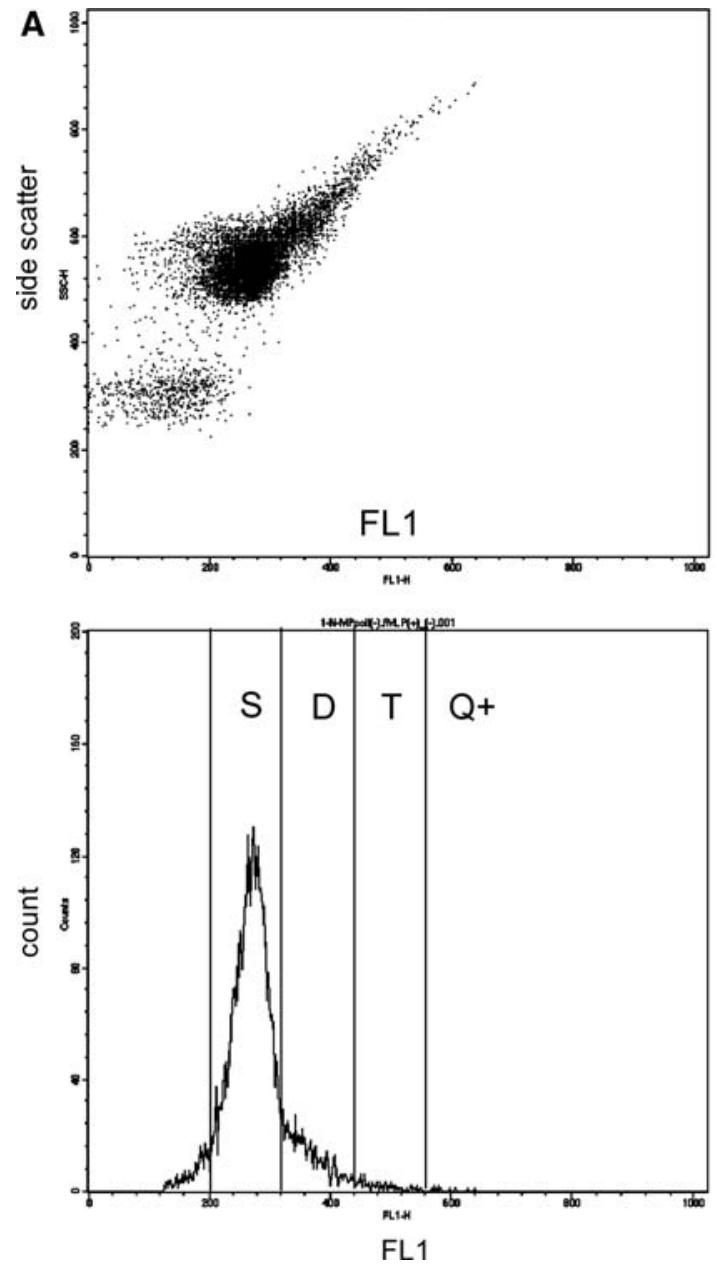

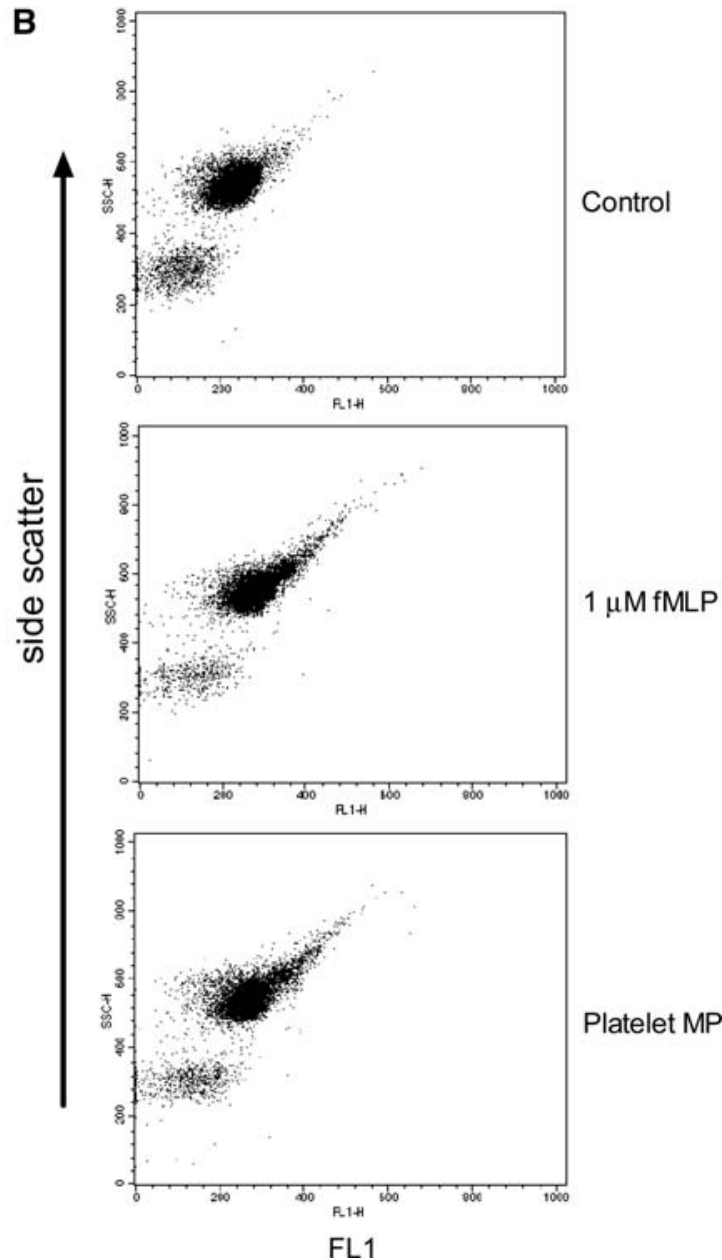

Figure 1. Platelet MP-supported neutrophil aggregation. Neutrophils were stirred at $700 \mathrm{rpm}$ in a platelet aggregometer for $15 \mathrm{~min}$ at $37{ }^{\circ} \mathrm{C}$ and were fixed with $2 \%$ glutaraldehyde for flow cytometry analysis. Determination of the neutrophil aggregation was described in Materials and methods. (A) Percentage of neutrophil aggregation calculated by use of the formula, \% aggregation $=\left(2 \mathrm{D}+3 \mathrm{~T}+4 \mathrm{Q}^{+}\right) /\left(\mathrm{S}+2 \mathrm{D}+3 \mathrm{~T}+4 \mathrm{Q}^{+}\right)$, where $\mathrm{S}$ represents singlet neutrophils and the neutrophil aggregate sizes are given as $\mathrm{D}$ (doublets), $\mathrm{T}$ (triplets), and $\mathrm{Q}^{+}$(quartets and larger unresolved neutrophil aggregates). (B) Neutrophil aggregate formation for unstimulated control (upper panel), after $1 \mu \mathrm{M}$ formyl-Met-Leu-Phe (fMLP) stimulation (middle panel), or platelet MP-stimulation (lower panel).

sediments obtained from PRP preparation were mixed with equal volume of platelet MP-containing plasma or PRP as control. The process of reconstitution was always performed within 90 min to minimize possible cell activation. For inhibitory study, inhibitors were added during the reconstitution process. In some preparations, high-speed centrifuged plasma (see above) substituted for platelet MP-containing plasma to confirm the effects of platelet MP. The reconstituted blood samples were then incubated for a further $30 \mathrm{~min}$ at $37^{\circ} \mathrm{C}$ under gentle agitation. After the incubation, $200 \mu \mathrm{l}$ of reconstituted whole blood samples were incubated for $30 \mathrm{~min}$ at $4{ }^{\circ} \mathrm{C}$ in the dark with appropriate FITC or PE-conjugated monoclonal antibodies (5 $\mu \mathrm{l}$ each). The samples were then fixed and red cells were lysed with FACS lysing solution according to the manufacture's instruction. After washing with PBS, they were resuspended in $500 \mu \mathrm{l}$ PBS containing 1\% formaldehyde and analyzed with a FACScan cytometer with the Cell Quest software (Becton Dickinson). The leukocytes were gated according to their forward and side scatter characteristics as well as by gating CD14 and CD11b positive cells. 


\section{Statistical analysis}

Results are presented as mean \pm SD. Statistical comparisons were made by ANOVA analysis followed by Dunn's test. Values of $p<0.05$ were considered significant for all tests.

\section{Results}

Involvement of GPIb in neutrophil aggregation supported by platelet MP

Neutrophil-neutrophil homotypic aggregation sequentially involves first L-selectin binding to PSGL-1 and then $\beta 2$ integrin and requires a threshold hydrodynamic shear greater than venous level [30]. It has been previously reported that platelet MP can support neutrophil aggregation under venous shear stress via P-selectin and PSGL-1 interaction. To further investigate the role of GPIb in the platelet MP-supported neutrophil aggregation, we studied the neutrophil aggregation in shear stress generated by stirring bar in an aggregometer and analyzed the aggregated neutrophil population by flow cytometry, as detailed in Methods and materials (Figure 1A). As shown in Figure 1B, the percentage of aggregation of unstimulated neutrophils was less than $5 \%$, while fMLP stimulation and platelet MPs enhanced neutrophil aggregation. EDTA totally abolished the fMLP-induced neutrophil aggregation (data not shown). $\beta 2$ integrin blocking $\mathrm{mAb}$ inhibited the fMLP-stimulated neutrophil aggregation by $\sim 55 \%$ (Figure $2 \mathrm{~A}$ ).

In the inhibitory experiments on the platelet PM-mediated neutrophil aggregation (Figure 2B), we found that P-selectin blocking $\mathrm{mAb} 9 \mathrm{E} 1$ inhibited aggregated neutrophil population by $\sim 45 \%$, and GPIb blocking $\mathrm{mAb}$ AP1 and SZ2 also significantly inhibited the level of neutrophil aggregation by $\sim 25 \%$. In contrast, GPIIb/IIIa antagonists, c7E3 or triflavin, had no significant inhibitory effect on platelet MP-mediated neutrophil aggregation. $\beta 2$ integrin $\mathrm{mAb}$ (anti-CD18, Ts1/18) failed to inhibit the neutrophil aggregation, indicating that platelet MP-mediated neutrophil aggregation depends largely on platelet P-selectin and GPIb, but not on $\beta 2$ integrin bridging mechanism.
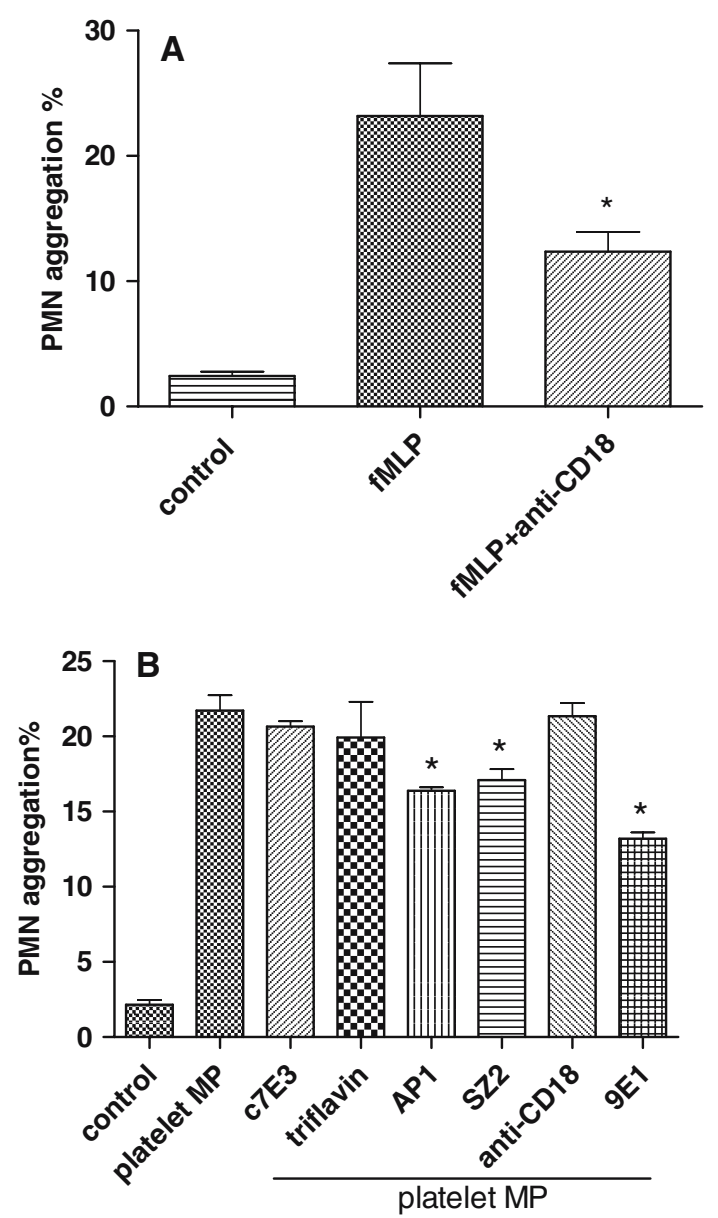

Figure 2. Inhibitory effects of GPIb inhibitors and P-selectin blocking antibody on platelet MP-stimulated neutrophil aggregate formation. Neutrophil isolates were stirred at $700 \mathrm{rpm}$ in a platelet aggregometer $15 \mathrm{~min}$ at $37^{\circ} \mathrm{C}$ and were fixed with $2 \%$ glutaraldehyde for flow cytometry analysis (A). Neutrophil isolates were stimulated with $1 \mu \mathrm{M}$ formyl-MetLeu-Phe (fMLP) with or without anti-CD $18(10 \mu \mathrm{g} / \mathrm{ml})$. Unstimulated neutrophils served as control. $* p<0.05$ vs. fMLP-stimulated neutrophils (B). Neutrophils were mixed with equal volume of platelet MP-containing plasma and stirred in aggregometer. For inhibitory studies, the following inhibitors were added into neutrophils $5 \mathrm{~min}$ before the addition of platelet MP-containing plasma: triflavin $(10 \mu \mathrm{g} / \mathrm{ml})$, c7E3 $(20 \mu \mathrm{g} / \mathrm{ml}), 9 \mathrm{E} 1(20 \mu \mathrm{g} / \mathrm{ml})$, AP1 $(20 \mu \mathrm{g} / \mathrm{ml})$, SZ2 $(20 \mu \mathrm{g} /$ $\mathrm{ml})$ or anti-CD18 $(10 \mu \mathrm{g} / \mathrm{ml}) .{ }^{*} p<0.05$ vs. platelet MP, $n=3$.

Involvement of GPIb in neutrophil ROS production induced by platelet $M P$

We next evaluated the effects of various inhibitors on the respiratory bursts by platelet MP-stimulated neutrophils utilizing an established microplate assay. Coincubation of neutrophils with 
platelet MP induced superoxide production at a similar level by neutrophils stimulated with $1 \mu \mathrm{M}$ fMLP. Since previous studies have demonstrated the involvement of $\beta 2$ integrin and tyrosine kinase in the production of superoxide from fMLPstimulated adherent neutrophils [31], we next examined the role of $\beta 2$ integrin and tyrosine kinase in the respiratory bursts of neutrophils treated with platelet MP-plasma. Anti-CD18 blocking $\mathrm{mAb}(10 \mu \mathrm{g} / \mathrm{ml})$ and tyrosine kinase inhibitor genistein $(5 \mu \mathrm{g} / \mathrm{ml})$ both effectively inhibited the superoxide production by platelet MP-stimulated neutrophils (Figure 3). Significant inhibition of the respiratory bursts was observed in the platelet MP-stimulated neutrophils in the presence of platelet GPIb inhibitors AP1 and SZ2.

\section{Involvement of GPIb in neutrophil adhesion induced by platelet MP}

As shown in Figure 4, platelet MP-promoted the neutrophils to adhere to fibrinogen with a similar level comparable to that induced by fMLP stimulation. About 50 and $30 \%$ of the adherence of the platelet MP-stimulated neutrophils were blocked by $\mathrm{mAb}$ anti-CD18 $(10 \mu \mathrm{g} / \mathrm{ml})$ and tyrosine kinase inhibitor genistein $(5 \mu \mathrm{g} / \mathrm{ml})$, suggesting that the

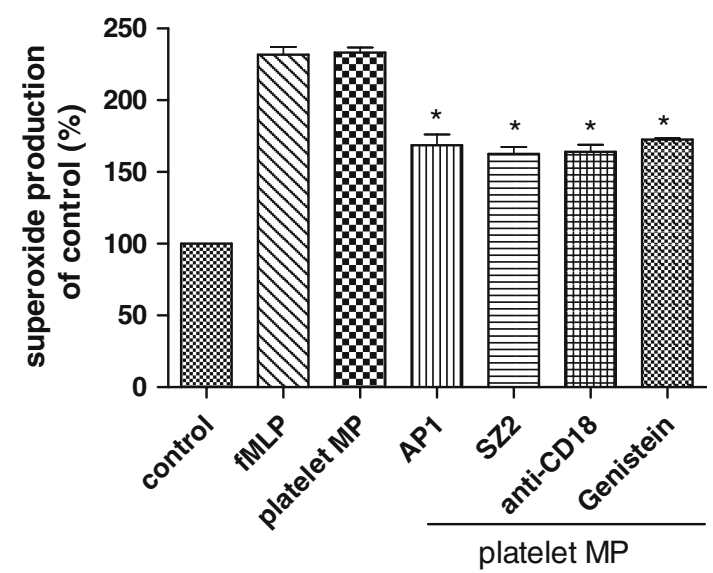

Figure 3. Neutrophil respiratory bursts after platelet MP-stimulation. Neutrophils $\left(1 \times 10^{5}\right)$ were added into fibrinogen-coated microwells in the presence of $1 \mu \mathrm{M}$ formylMet-Leu-Phe (fMLP) or $100 \mu \mathrm{l}$ platelet MP-containing plasma. Various inhibitors were added for inhibitory study: AP1 $(20 \mu \mathrm{g} / \mathrm{ml})$, SZ2 $(20 \mu \mathrm{g} / \mathrm{ml})$, anti-CD18 $(10 \mu \mathrm{g} / \mathrm{ml})$ and genistein $(5 \mu \mathrm{g} / \mathrm{ml})$. Respiratory bursts were performed by a cytochrome $c$ reduction method. Data were presented as percentage vs. unstimulated neutrophil control $\left({ }^{*} p<0.05\right.$ vs. platelet MP, $n=3$ ).

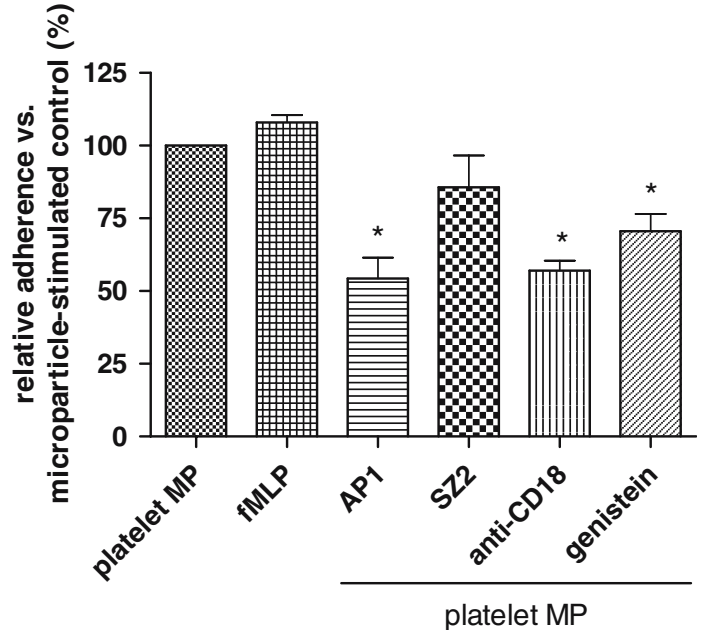

Figure 4. Platelet MP-promoted neutrophil adherence to immobilized fibrinogen. BCECF-AM loaded-neutrophils $\left(1 \times 10^{5}\right)$ were added into fibrinogen-coated microwells in the presence of $1 \mu \mathrm{M}$ formyl-Met-Leu-Phe (fMLP) or $100 \mu \mathrm{l}$ platelet MP-containing plasma. Various inhibitors were added: AP1 $(20 \mu \mathrm{g} / \mathrm{ml})$, SZ2 $(20 \mu \mathrm{g} / \mathrm{ml})$, anti-CD18 $(10 \mu \mathrm{g} / \mathrm{ml})$ and genistein $(5 \mu \mathrm{g} / \mathrm{ml})$. After $30 \mathrm{~min}$ incubation at $37^{\circ} \mathrm{C}$, microplates were washed extensively, and coated cells were solubilized and fluorescence was read by a Cytofluor microplate reader. Data are mean $\pm \mathrm{SD}$ of three independent experiments $\left({ }^{*} p<0.05\right.$ vs. platelet MP, $\left.n=3\right)$.

involvement of $\beta 2$ integrin and tyrosine kinase in mediating neutrophil adherence-induced by platelet MP. Platelet GPIb blocking mAb AP1 $(20 \mu \mathrm{g} /$ $\mathrm{ml}$ ) effectively inhibited the adherence by $\sim 50 \%$, while SZ2 $(20 \mu \mathrm{g} / \mathrm{ml})$ failed to demonstrate statistically significant inhibition on the neutrophil adherence.

\section{Leukocyte activation after mixing with platelet $M P$ in reconstituted whole blood}

To investigate the interaction of platelet MP and leukocytes in a simulated whole blood system, we reconstituted whole blood samples by mixing platelet MP-containing plasma and blood cell sediments. The reconstituted whole blood samples were stained with PE or FITC-conjugated mAbs against CD42a (against platelet GPIX), CD62p (against P-selectin) or CD11b (against Mac-1) for flow cytometric assay (Figure 5). Neutrophils in the reconstituted whole blood showed increases in CD 42a- and CD62p-bound levels (\%) and CD11b expression $(\%)$, demonstrating that platelet MPs-transferred platelet-related antigens and subsequently stimulated neutrophils after the 

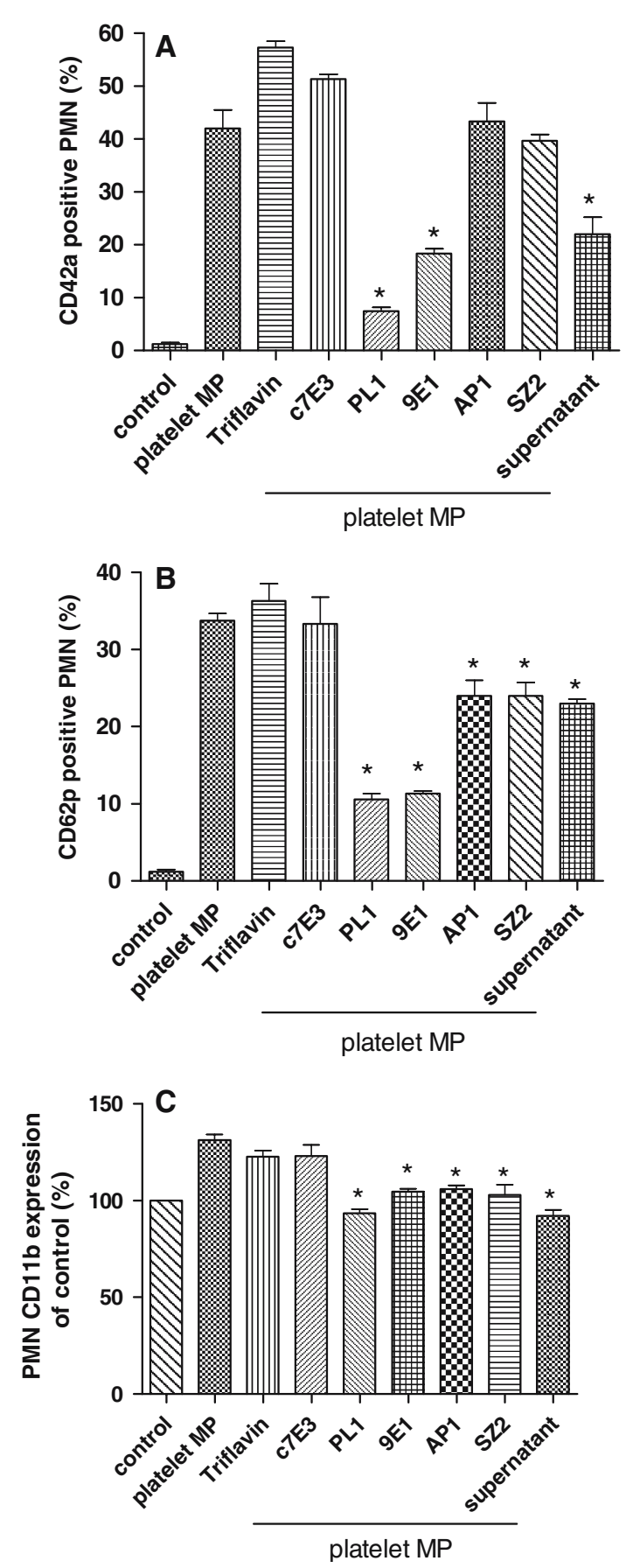

reconstitution of blood samples. High-speed centrifugation partially removed the plateletrelated antigens in the microparticles-containing plasma (Figure 5A, B) and abolished the stimulatory effect of platelet MP on neutrophil CD11b expression (Figure 5C).
Figure 5. Neutrophil-bound platelet antigen expression and CD11b expression with reconstituted experiments. Platelet MP-containing plasma or platelet-rich plasma (PRP) as control was mixed with cell sediments obtained after the preparation of platelet-rich plasma (see Materials and methods) in equal volume, the reconstituted blood samples were stained with FITC or PE-conjugated anti-CD42a, CD62p or CD11b $\mathrm{mAbs}$, and were analyzed with flow cytometry. In inhibitory experiments, various inhibitors were added during the reconstitution process: Triflavin $(10 \mu \mathrm{g} / \mathrm{ml})$, c7E3 $(20 \mu \mathrm{g} / \mathrm{ml})$, PL1 $(20 \mu \mathrm{g} / \mathrm{ml}), 9 \mathrm{E} 1(20 \mu \mathrm{g} / \mathrm{ml})$ AP1 $(20 \mu \mathrm{g} / \mathrm{ml})$, or SZ2 $(20 \mu \mathrm{g} /$ $\mathrm{ml})$. For confirmation of the effect of MP, platelet MP-containing plasma was subsequently centrifuged $30 \mathrm{~min}$ at $17,500 \mathrm{~g}$ to obtain the supernatant for removal of microparticles. Percentage of CD42a-positive PMN (A), CD62P-positive PMN (B) and CD11b-positive PMN (C) were shown $\left({ }^{*} p<0.05\right.$ vs. platelet MP, $\left.n=3\right)$.

To clarify the effects of inhibitory $\mathrm{mAb}$ or protein on platelet-MP and neutrophil interaction, various inhibitors were added while reconstituting the whole blood. PL1 (a blocking mAb against human PSGL-1 at $20 \mu \mathrm{g} / \mathrm{ml}$ ) and 9E1 (a blocking $\mathrm{mAb}$ against human P-selectin at $20 \mu \mathrm{g} / \mathrm{ml}$ ) markedly reduced the levels of platelet-antigens bound on neutrophils, and abolished the increased expression of CD11b on neutrophils. The percentages of neutrophils positive for CD62p were significantly reduced in the presence of two platelet GPIb blocking mAbs, AP1, and SZ2 while the percentages of CD42a bound neutrophils did not change with the treatment of AP1 or SZ2. Both antibodies blocked the increased neutrophil $\mathrm{CD} 11 \mathrm{~b}$ expression in the reconstitution blood.

We next examined the inhibitory effects of two platelet GPIIb/IIIa blocking agents, mAb c7E3 and triflavin, on the platelet-MP and neutrophil interaction in reconstituted blood experiment. These GPIIb/IIIa inhibitors did inhibit plateletantigens transfer and the subsequent enhanced CD11b expression on neutrophils. Paradoxically, triflavin increased CD42a-bound neutrophil population in the reconstituted blood. Similar effects of GPIIb/IIIa inhibitors on platelet-leukocyte interaction have been observed by us (unpublished data) and others $[32,33]$.

\section{Discussion}

The scenario of platelet-leukocyte interaction involves a cascade of interaction of various adhesion molecules. The initial contact corresponding to the tethering of platelets on the surface of 
neutrophils and monocytes is mainly mediated by the interaction of platelet P-selectin with PSGL-1, which is constitutively expressed by leukocytes. In this setting, only the activated platelets are able to adhere to the neutrophils and monocytes. Firm adherence of platelets to neutrophils is then maintained by fibrinogen bridging, which couples the platelet GPIIb/IIIa to $\beta 2$ integrin (i.e. CD11b/ 18). Further adhesion partners of Mac-1 on the activated platelet cell membrane are GPIb [8] and Jam-3 [34]. Recently, Simon and his colleagues further identified $\alpha$ MI domain of Mac-1 as the binding site for platelet glycoprotein Ib $\alpha$ and established the crucial role of GPIb-Mac-1 engagement for the biological response in vascular injury [35].

Our study suggests that, like the interaction between platelet and leukocyte, platelet MPs initiates signal transduction and activation of neutrophils via P-selectin binding to PSGL-1 on neutrophils. In addition to P-selectin/PSGL-1 interaction, platelet MPs also activate neutrophils through interaction of GPIb with activated Mac-1. These interactions induce neutrophil activation, as shown by the increased neutrophil ROS production and adhesion to fibrinogen (Figures 3 and 4). Our data also showed that tyrosine kinase is likely involved in the platelet MPs-induced neutrophil activation, which is in accordance with a previous report that activation of neutrophil mediated by P-selectin involves tyrosine kinase activation [31].

Neutrophil aggregation contributes to the recruitment of inflammatory cells and to the pathophysiological obstruction of vessels. Guyer et al. previously observed that homotypic neutrophil aggregation requires two types of molecular interaction [36]: (1) a transient constitutive interaction between L-selectin and a carbohydratebearing structure; and (2) after stimulation, a high affinity interaction that mediates leukocyte adhesion to endothelial cells. In this model, L-selectin first recognizes its glycosylated ligand, and then the activated integrin binds tightly to its counterstructure, possibly an ICAM family member [37-40]. Our data showed that platelet MPs can support neutrophil aggregate under venous level of shear stress, and platelet MPs bridge neutrophils via P-selectin and GPIb (Figure 2B).

Two mechanisms may be involved in the neutrophil aggregation mediated by platelet MPs: namely, (1) by platelet MPs bridging between neutrophils and (2) through contact-mediated activation. Our study showed that $\beta 2$ integrin blockade with CD18 mAb virtually abolished neutrophil homotypic aggregate after fMLP stimulation, but failed to reduce neutrophil aggregates triggered by platelet MPs (Figure 2B). This indicates that platelet MPs support neutrophil aggregate mainly by bridging neutrophils through platelet P-selectin and GPIb. It is feasible to reason that the activated state of neutrophils after the contact with platelet MPs do not support neutrophil homotypic aggregation. Ma et al. [41] have demonstrated that P-selectin binding can induce an intermediate state of $\beta 2$ integrin activation and must act cooperatively with extracellular stimuli to support maximal adhesion of neutrophils. Previous study has established the requirement of a shear threshold higher than venous shear stress for neutrophil-neutrophil interaction via L-selectin [30]. The finding that platelet MP can support neutrophil aggregation in venous shear stress may has significant clinical implications. As the blood concentration of platelet MPs is elevated in certain patients, platelet MPs may amplify leukocyte interaction, therefore promoting leukocyte-mediated tissue injury in thrombotic and inflammation disorder.

However, there are some limitations regarding our study of platelet MPs interacting with neutrophils. Mechanisms other than the contact interaction via P-selectin or GPIb may also contribute to the activation of monocytic cells after platelet MP exposure. Based on the result that P-selectin and GPIb blockade consistently exerted inhibitory effects on the interaction of platelet MP with neutrophils in all our study, it seems that the contact mechanism plays a major role. Another possible limitation is that our study on neutrophil aggregation was performed in a low-shear stress mimicking venous levels of shear. It is well known that shear stress markedly affects GPIb interaction with its ligands. Observations in platelet aggregation showed that high-shear stress spontaneously induced GPIb to interact with von Willebrand factor in the absence of chemical agonists [42]. Hu et al. [43] further reported that differential involvement of selectins and integrins in platelet-leukocyte aggregation under shear stress. Further studies are needed to clarify the effect of shear stress on the platelet-MP-neutrophil interaction. 
In the study of neutrophil adhesion to fibrinogen, mAb AP1 significantly inhibited the adhesion, while $\mathrm{mAb}$ SZ2 only showed a trend to inhibit the adhesion but failed to reach a statistically significant level (Figure 4). In regard to the platelet MPs in promoting neutrophil adherence to fibrinogen, two mechanisms may be involved: neutrophil is activated by platelet MP then binds to fibrinogen via activated $\beta 2$ integrin [44] or platelet MP acts as a bridge between neutrophil and fibrinogen. Since AP1 and SZ2 both, to the similar extent, inhibited neutrophil activation by platelet MPs (Figure 5), it is likely that AP1 and SZ2 performed differently in bridging between neutrophil and fibrinogen. The epitope mapping may explain for their different bridging effectiveness. AP1 binds to the outside $\mathrm{COOH}$-terminal flanking and leucine-rich repeat region of $\mathrm{GPIb}$ (amino acid 201-268), while SZ2 maps to the sulfated tyrosine residues encompassing amino acids 268-282, which is nearer to the platelet membrane $[8,45]$. However, the exact mode of action needs further investigation.

In our study, we failed to demonstrate the involvement of GPIIb/IIIa in platelet MP-neutrophil interaction. Accordingly, many studies also have shown that GPIIb/IIIa blockade do not reduce platelet-neutrophil aggregation $[32,33]$.

In conclusion, our data provide the evidence for the involvement of GPIb-Mac-1 interaction in the cross-talk between platelet MPs and neutrophils, in addition to the well-established P-selectin/ PSGL-1 interaction. Our data raise the possibilities of targeting GPIb and/or P-selectin to prevent the pathological platelet MP-neutrophil interactions in inflammatory and/or thrombotic disorders.

\section{References}

1. Evangelista V., Manarini S., Sideri R., Rotondo S., Martelli N., Piccoli A., Totani L., Piccardoni P., Vestweber D., de Gaetano G. and Cerletti C., Platelet/polymorphonuclear leukocyte interaction: P-selectin triggers protein-tyrosine phosphorylation-dependent CD11b/CD18 adhesion: role of PSGL-1 as a signaling molecule. Blood 93: 876-885, 1999.

2. Nagata K., Tsuji T., Todoroki N., Katagiri Y., Tanoue K., Yamazaki H., Hanai N. and Irimura T., Activated platelets induce superoxide anion release by monocytes and neutrophils through P-selectin (CD62). J. Immunol. 151: 3267-3273, 1993.
3. McGarrity S.T., Stephenson A.H. and Webster R.O., Regulation of human neutrophil functions by adenine nucleotides. J. Immunol. 142: 1986-1994, 1989.

4. Tzeng D.Y., Deuel T.F., Huang J.S., Senior R.M., Boxer L.A. and Baehner R.L., Platelet-derived growth factor promotes polymorphonuclear leukocyte activation. Blood 64: 1123-1128, 1984.

5. Bebawy S.T., Gorka J., Hyers T.M. and Webster R.O., In vitro effects of platelet factor 4 on normal human neutrophil functions. J. Leukoc. Biol. 39: 423-434, 1986.

6. Mazolewski P.J., Roth A.C., Suchy H., Stephenson L.L. and Zamboni W.A., Role of the thromboxane A2 receptor in the vasoactive response to ischemia-reperfusion injury. Plast. Reconstr. Surg. 104: 1393-1396, 1999.

7. Barry O.P. and FitzGerald G.A., Mechanisms of cellular activation by platelet microparticles. Thromb. Haemost. 82: 794-800, 1999.

8. Simon D.I., Chen Z., Xu H., Li C.Q., Dong J., McIntire L.V., Ballantyne C.M., Zhang L., Furman M.I., Berndt M.C. and Lopez J.A., Platelet glycoprotein ibalpha is a counter-receptor for the leukocyte integrin Mac-1 (CD11b/ CD18). J. Exp. Med. 192: 193-204, 2000.

9. Weber C. and Springer T.A., Neutrophil accumulation on activated, surface-adherent platelets in flow is mediated by interaction of Mac-1 with fibrinogen bound to alphaIIbbeta3 and stimulated by platelet-activating factor. J. Clin. Invest. 100: 2085-2093, 1997.

10. Yang J., Furie B.C. and Furie B., The biology of P-selectin glycoprotein ligand-1: its role as a selectin counter-receptor in leukocyte-endothelial and leukocyte-platelet interaction. Thromb. Haemost. 81: 1-7, 1999.

11. Palabrica T., Lobb R., Furie B.C., Aronovitz M., Benjamin C., Hsu Y.M., Sajer S.A. and Furie B., Leukocyte accumulation promoting fibrin deposition is mediated in vivo by P-selectin on adherent platelets. Nature 359: 848851, 1992.

12. Tan K.T. and Lip G.Y., The potential role of platelet microparticles in atherosclerosis. Thromb. Haemost. 94: 488-492, 2005.

13. Joop K., Berckmans R.J., Nieuwland R., Berkhout J., Romijn F.P., Hack C.E. and Sturk A., Microparticles from patients with multiple organ dysfunction syndrome and sepsis support coagulation through multiple mechanisms. Thromb. Haemost. 85: 810-820, 2001.

14. Sarma J., Laan C.A., Alam S., Jha A., Fox K.A. and Dransfield I., Increased platelet binding to circulating monocytes in acute coronary syndromes. Circulation 105: 2166-2171, 2002.

15. Furman M.I., Barnard M.R., Krueger L.A., Fox M.L., Shilale E.A., Lessard D.M., Marchese P., Frelinger A.L. III, Goldberg R.J. and Michelson A.D., Circulating monocyte-platelet aggregates are an early marker of acute myocardial infarction. J. Am. Coll. Car. Diol. 38: 10021006, 2001.

16. Michelson A.D., Barnard M.R., Krueger L.A., Valeri C.R. and Furman M.I., Circulating monocyte-platelet aggregates are a more sensitive marker of in vivo platelet activation than platelet surface P-selectin: studies in baboons, human coronary intervention, and human acute myocardial infarction. Circulation 104: 1533-1537, 2001.

17. Randomised placebo-controlled trial of abciximab before and during coronary intervention in refractory unstable angina: the CAPTURE Study. Lancet 349: 1429-1435, 1997. 
18. McCabe D.J., Harrison P., Mackie I.J., Sidhu P.S., Purdy G., Lawrie A.S., Watt H., Brown M.M. and Machin S.J., Platelet degranulation and monocyte-platelet complex formation are increased in the acute and convalescent phases after ischaemic stroke or transient ischaemic attack. Br. J. Haematol. 125: 777-787, 2004.

19. Diamant M., Nieuwland R., Pablo R.F., Sturk A., Smit J.W. and Radder J.K., Elevated numbers of tissue-factor exposing microparticles correlate with components of the metabolic syndrome in uncomplicated type 2 diabetes mellitus. Circulation 106: 2442-2447, 2002.

20. Dachary-Prigent J., Pasquet J.M., Fressinaud E., Toti F., Freyssinet J.M. and Nurden A.T., Aminophospholipid exposure, microvesiculation and abnormal protein tyrosine phosphorylation in the platelets of a patient with Scott syndrome: a study using physiologic agonists and local anaesthetics. Br. J. Haematol. 99: 959-967, 1997.

21. Jy W., Mao W.W., Horstman L., Tao J. and Ahn Y.S., Platelet microparticles bind, activate and aggregate neutrophils in vitro. Blood Cells Mol. Dis. 21: 217-231, 1995.

22. Sheu J.R. and Huang T.F., Triflavin, an Arg-Gly-Aspcontaining peptide, inhibits B16-F10 mouse melanoma cell adhesion to matrix proteins via direct binding to tumor cells. J. Biomed. Sci. 3: 359-364, 1996.

23. Huang T.F., Sheu J.R. and Teng C.M., Mechanism of action of a potent antiplatelet peptide, triflavin from Trimeresurus flavoviridis snake venom. Thromb. Haemost. 66: 489-493, 1991.

24. Tseng Y.L., Lee C.J. and Huang T.F., Effects of a snake venom metalloproteinase, triflamp, on platelet aggregation, platelet-neutrophil and neutrophil-neutrophil interactions: involvement of platelet GPIbalpha and neutrophil PSGL-1. Thromb. Haemost. 91: 315-324, 2004.

25. Khreiss T., Jozsef L., Potempa L.A. and Filep J.G., Loss of pentameric symmetry in C-reactive protein induces interleukin-8 secretion through peroxynitrite signaling in human neutrophils. Circ. Res. 97: 690-697, 2005.

26. Konstantopoulos K., Neelamegham S., Burns A.R., Hentzen E., Kansas G.S., Snapp K.R., Berg E.L., Hellums J.D., Smith C.W., McIntire L.V. and Simon S.I., Venous levels of shear support neutrophil-platelet adhesion and neutrophil aggregation in blood via P-selectin and beta2-integrin. Circulation 98: 873-882, 1998.

27. Simon S.I., Burns A.R., Taylor A.D., Gopalan P.K., Lynam E.B., Sklar L.A. and Smith C.W., L-selectin (CD62L) cross-linking signals neutrophil adhesive functions via the Mac-1 (CD11b/CD18) beta 2-integrin. J. Immunol. 155: 1502-1514, 1995.

28. Tseng Y.L., Lee C.J., Hsu C.C. and Huang T.F., Triflamp, a snake venom metalloproteinase, reduces neutrophil-platelet adhesion through proteolysis of PSGL-1 but not glycoprotein Ib alpha. Thromb. Haemost. 91: 1177-1185, 2004.

29. Scholz T., Temmler U., Krause S., Heptinstall S. and Losche W., Transfer of tissue factor from platelets to monocytes: role of platelet-derived microvesicles and CD62P. Thromb. Haemost. 88: 1033-1038, 2002.

30. Finger E.B., Puri K.D., Alon R., Lawrence M.B., von Andrian U.H. and Springer T.A., Adhesion through L-selectin requires a threshold hydrodynamic shear. Nature 379: 266-269, 1996.

31. Hidari K.I., Weyrich A.S., Zimmerman G.A. and McEver R.P., Engagement of P-selectin glycoprotein ligand-1 enhances tyrosine phosphorylation and activates mitogenactivated protein kinases in human neutrophils. J. Biol. Chem. 272: 28750-28756, 1997.

32. Zhao L., Bath P.M., Fox S., May J., Judge H., Losche W. and Heptinstall S., The effects of GPIIb-IIIa antagonists and a combination of three other antiplatelet agents on platelet-leukocyte interactions. Curr. Med. Res. Opin. 19: 178-186, 2003.

33. Zhao L., Bath P.M., May J., Losche W. and Heptinstall S., P-selectin, tissue factor and CD40 ligand expression on platelet-leucocyte conjugates in the presence of a GPIIb/ IIIa antagonist. Platelets 14: 473-480, 2003.

34. Santoso S., Sachs U.J., Kroll H., Linder M., Ruf A., Preissner K.T. and Chavakis T., The junctional adhesion molecule 3 (JAM-3) on human platelets is a counterreceptor for the leukocyte integrin Mac-1. J. Exp. Med. 196: 679-691, 2002.

35. Shibamiya A., Tabuchi N., Chung J., Sunamori M. and Koyama T., Formation of tissue factor-bearing leukocytes during and after cardiopulmonary bypass. Thromb. Haemost. 92: 124-131, 2004.

36. Guyer D.A., Moore K.L., Lynam E.B., Schammel C.M., Rogelj S., McEver R.P. and Sklar L.A., P-selectin glycoprotein ligand-1 (PSGL-1) is a ligand for L-selectin in neutrophil aggregation. Blood 88: 2415-2421, 1996.

37. Lynam E.B., Simon S.I., Rochon Y.P. and Sklar L.A., Lipopolysaccharide enhances $\mathrm{CD} 11 \mathrm{~b} / \mathrm{CD} 18$ function but inhibits neutrophil aggregation. Blood 83: 3303-3311, 1994.

38. Bennett T.A., Schammel C.M., Lynam E.B., Guyer D.A., Mellors A., Edwards B., Rogelj S. and Sklar L.A., Evidence for a third component in neutrophil aggregation: potential roles of O-linked glycoproteins as L-selectin counterstructures. J. Leukoc. Biol. 58: 510-518, 1995.

39. Simon S.I., Rochon Y.P., Lynam E.B., Smith C.W., Anderson D.C. and Sklar L.A., Beta 2-integrin and L-selectin are obligatory receptors in neutrophil aggregation. Blood 82: 1097-1106, 1993.

40. Rochon Y.P., Simon S.I., Lynam E.B. and Sklar L.A., A role for lectin interactions during human neutrophil aggregation. J. Immunol. 152: 1385-1393, 1994.

41. Ma Y.Q., Plow E.F. and Geng J.G., P-selectin binding to P-selectin glycoprotein ligand-1 induces an intermediate state of alphaMbeta2 activation and acts cooperatively with extracellular stimuli to support maximal adhesion of human neutrophils. Blood 104: 2549-255, 2004.

42. Fredrickson B.J., Dong J.F., McIntire L.V. and Lopez J.A., Shear-dependent rolling on von Willebrand factor of mammalian cells expressing the platelet glycoprotein IbIX-V complex. Blood 92: 3684-3693, 1998.

43. Biro E., Sturk-Maquelin K.N., Vogel G.M., Meuleman D.G., Smit M.J., Hack C.E., Sturk A. and Nieuwland R., Human cell-derived microparticles promote thrombus formation in vivo in a tissue factor-dependent manner. J. Thromb. Haemost. 1: 2561-2568, 2003.

44. Ugarova T.P. and Yakubenko V.P., Recognition of fibrinogen by leukocyte integrins. Ann. N.Y. Acad. Sci. 936: 368-385, 2001

45. Berndt M.C., Du X.P. and Booth W.J., Ristocetin-dependent reconstitution of binding of von Willebrand factor to purified human platelet membrane glycoprotein Ib-IX complex. Biochemistry 27: 633-640, 1988. 